\title{
DIOPHANTINE AND COHOMOLOGICAL DIMENSIONS
}

\author{
DANIEL KRASHEN AND ELIYAHU MATZRI
}

\begin{abstract}
Aвstract. We give explicit linear bounds on the $p$-cohomological dimension of a field in terms of its Diophantine dimension. In particular, we show that for a field of Diophantine dimension at most 4, the 3 -cohomological dimension is less than or equal to the Diophantine dimension.
\end{abstract}

\section{INTRODUCTION}

Let $F$ be a field and suppose that $p$ is a prime integer not equal to the characteristic of $F$. Recall that a field $F$ is said to have the $C_{d}$ property if every homogeneous polynomial of degree $n$ over $F$ in more than $n^{d}$ variables has a nontrivial zero. The Diophantine dimension of $F$, denoted $\operatorname{ddim}(F)$ is defined to be the least integer $d$ such that $F$ has the $C_{d}$ property. We define the $p$-cohomological dimension of $F$, denoted $\operatorname{cd}_{p}(F)$ to be the least integer $d$ such that the Galois cohomology groups $H^{d}(L, \mathbb{Z} / p \mathbb{Z})$ vanish for every $L / F$ finite. One says that $F$ has cohomological dimension $d$, written $\operatorname{cd}(F)=d$ if $d$ is the maximum value of $\operatorname{cd}_{p}(F)$ taken over all $p$.

Although there are well known examples of Ax [Ax65] of fields of cohomological dimension 1 which do not have the $C_{d}$ property for any $d$, it is quite possible that having a bound on the Diophantine dimension will give one a bound on the $p$-cohomological dimension. In [Ser94, page 99], after outlining why the Milnor Conjectures would imply $\operatorname{cd}_{2}(F) \leq \operatorname{ddim}(F)$, Serre states "Il est probable que ce résultat est également valable pour $p \neq 2$." On the other hand, is was not known until present whether or not a field with $\operatorname{ddim}(F)$ finite must have $\operatorname{cd}_{p}(F)$ finite in general for $p \neq 2$.

In this paper we give explicit bounds on the $p$-cohomological dimensions in terms of the Diophantine dimension, and show in particular that $\operatorname{cd}_{p}(F)$ grows at most linearly with respect to $\operatorname{ddim}(F)$ (see Theorem 1.15).

Throughout, we let $p$ be a prime number and use the notation $K_{i}^{M}(F)$ to denote the $i^{\prime}$ th Milnor K-group of $F$, and $k_{i}(F)=K_{i}^{M}(F) / p K_{i}^{M}(F)$. By the Bloch-Kato conjecture, we may identify $k_{i}(F)=H^{i}\left(F, \mu_{p}^{\otimes i}\right)$. We will use the notation $\left(a_{1}, \ldots, a_{n}\right)$ to denote a symbol in the group $k_{n}(F)$. In the case that $\rho \in F$ is a primitive $p^{\prime}$ th root of unity, we will use the notation $D_{(a, b)}$

The first author was partially supported by NSF grants DMS-1007462 and DMS-1151252.

The second author was partially supported by the Kreitman foundation. 
to denote the symbol algebra generated by elements $x, y$ and satisfying $x^{p}=a, y^{p}=b, x y=\rho y x$.

Acknowledgments. The authors would like to thank Skip Garibaldi for useful conversations, and help with finding references during the writing of this manuscript.

\section{Homogeneous forms And Milnor K-Theory}

Definition 1.1. By a form of degree $d$ and dimension $n$, we mean a homogeneous degree $d$ polynomial function $f \in F\left[V^{*}\right]$ where $V$ is an $n$-dimensional vector space. We will also refer to $f$ as a degree $d$ form on $V$. We say that $f$ is isotropic if there is a $v \in V \backslash\{0\}$ such that $f(v)=0$.

Definition 1.2. Suppose that $\alpha \in k_{n}(F)$, and let $V$ be a vector space. We say that a form $N$ on $V$

- neutralizes $\alpha$ if $\alpha \cup(N(v))$ is trivial in $k_{n}(L)$ for every field extension $L / F$ and every choice of $v \in V_{L}$ with $N(v) \neq 0$,

- splits $\alpha$ if for every field extension $L / F$, we have $N_{L}$ isotropic implies $\alpha_{L}=0$.

Definition 1.3. Let $A$ be a unital finite dimensional strictly power associative algebra, and let $N_{A} \in F\left[A^{*}\right]$ be the reduced norm form (see Section 2). As in Section 2, we say that $A$ is principally division, if for every $a \in A$, the subalgebra $F[a]$ generated by $a$ is a division algebra. We say that $A$ is adapted to a class $\alpha \in k_{n}(F)$ if $N_{A}$ is degree $p$ and if for every field extension $L / F, A_{L}$ is principally division if and only if $\alpha_{L} \neq 0$.

Lemma 1.4. Suppose that $A$ is a unital finite dimensional strictly power associative algebra which is adapted to $\alpha \in k_{n}(F)$. Then $N_{A}$ neutralizes and splits $\alpha$.

Proof. Let $d=N_{A}(x)$ for $x \in A$. We claim that $\alpha \cup(d)=0$. If $x \in F \subset A$ then $N_{A}(x)=x^{p}=d$ and consequently $\alpha \cup(d)=p \alpha \cup(x)=0$. On the other hand, if $x \in A \backslash F$ then $F(x)$ is a degree $p$ subfield of $A$ which by assumption concerning the characteristic of $F$ is separable. After passing to a prime-to- $p$ extension, which will not affect the triviality of $\alpha \cup(d)$ due to the standard restriction-corestriction argument, we may in fact assume that $F(x) / F$ is a cyclic Kummer extension, say $E=F(x)=F(y)$ where $y \in A$ with $y^{d}=b \in F$.

Now, $A_{E}$ contains $E \otimes E$ which contains zerodivisors. Since $E / F$ is separable, $E$ is principally generated over $F$, say $E=F[a]$, and hence $E \otimes E=E[a \otimes 1]$ is also principally generated. It follows that $A_{E}$ is not principally division. Since $N_{A}$ is adapted to $\alpha$, this implies in turn that $\alpha_{E}$ is split. But now, by [LMS07, Theorem 5] (see also [Voe03, Proposition 5.2]), we have the exact sequence:

$$
k_{n-1}(E) \stackrel{N_{E / F}}{\longrightarrow} k_{n-1}(F) \stackrel{\cup(b)}{\longrightarrow} k_{n}(F) \longrightarrow k_{n}(E)
$$

which tells us that $\alpha=\beta \cup(b)$ for some $\beta \in k_{n-1}(F)$. But now, we claim that $\alpha \cup(d)=0$. In fact this will come from the fact that $(b) \cup(d)=(b, d)=0$. Again, 
by the exact sequence above, this amounts to saying that $d \in N_{E / F}\left(k_{1}(E)\right)=$ $N_{E / F}\left(E^{*}\right)$. But $d=N_{E / F}(x), x \in E^{*}$ by hypothesis.

Next, suppose that $N_{A}(x)=0$, we need to show that $\alpha=0$ in this case. We will do this by showing that $A$ is not principally division, which would imply $\alpha=0$ by our hypothesis on $A$.

Arguing by contradiction, suppose $A$ is principally division. Since $\left.N_{A}\right|_{F}$ is the $p^{\prime}$ th power operation on $F$, and $F$ is a field, it follows that $x \in A \backslash F$ and hence $F(x)=E$ is a degree $p$ separable field extension. Since $x$ satisfies its characteristic polynomial $\chi_{x}(T)$ of degree $p$, it follows that $\chi_{x}(T)$ is in fact the minimal polynomial of $x$ as an element of $E$. Since $E$ is a field, $\chi_{x}(T)$ must be irreducible. But, since $N_{A}(x)=0$ is the constant coefficient of this polynomial, we find that $\chi_{x}(T)=T f(T)$ for some monic polynomial $f(T)$ contradicting the irreducibility of $\chi_{x}(T)$.

Example 1.5 (Kummer extensions). If $L=F(\sqrt[p]{a})$. Then $L$ is adapted to (a). This follows from the fact that $L / F$ is a division algebra exactly when $a \notin\left(F^{*}\right)^{p}$, which in turn happens exactly when $(a) \neq 0$ in $k_{1}\left(F^{*}\right)$.

Example 1.6 (Symbol algebras). Assume $\mu_{p} \subset F$, and let $\alpha=(a, b) \in k_{2}(F)=$ $\operatorname{Br}(F)_{p}$. Let $D=D_{(a, b)}$ be the corresponding symbol algebra. Then $D$ is adapted to $\alpha$. Indeed, since $D$ has degree $p$, it is either a split algebra or a division algebra. Further, it is split if and only if $(a, b)=0 \in k_{2}(F)=\operatorname{Br}(F)_{p}$, and it is division if and only if its norm form is anisotropic, and as we see in Lemma 2.2, it is division if and only if it is principally division.

Example 1.7 (First Tits process Albert algebras). Consider the case $p=$, suppose that $\mu_{3} \in F$ and let $D=D_{(a, b)}$ be the symbol algebra as before. Choose $c \in F^{*}$ and let $A=D_{0} \oplus D_{1} \oplus D_{2}$ with $D_{i}=D$ be the Albert algebra given by the first Tits process (see, for example [PR96, section 2.5], or [KMRT98, Section IX.39]).

We claim that $A$ is adapted to $(a, b, c)$. This follows from [PR96, Theorem 1.8 and Proposition 2.6], where it is shown that $A$ is division if and only if $(a, b, c) \neq 0$, and Lemma 2.3. where we show that $A$ is principally division if and only if it is division.

We will have use of the following identity in $k_{2}(F)$

Lemma 1.8. Suppose $a, b \in F^{*}$ with $a+b \neq 0$. Then $(a, b)=\left(-a b^{-1}, a+b\right)=$ $\left(a+b,-b a^{-1}\right)$.

Proof. Since passing to a prime-to- $p$ extension constitutes an injective map on the level of $k_{2}$, we may assume that $\mu_{p} \subset F$, and in particular, we may identify $k_{2}(F)=\operatorname{Br}(F)_{p}$ by the Merkurjev-Suslin Theorem. In particular, it suffices to show that $D_{(a, b)} \cong D_{\left(a+b,-a b^{-1}\right)}$. We do this as follows: presenting $D_{(a, b)}$ as generated by $x, y$ with $x^{p}=a, y^{p}=b, y x=\rho x y$, we let $z=x+$ $y$ and $w=-x y^{-1}$, we see $z^{p}=x^{p}+y^{p}=a+b, w^{p}=\mathrm{N}_{F[x] / F}(-x) b^{-1}=$ 


$$
\begin{aligned}
& (-1)^{p}(-1)^{p-1} a b^{-1}=-a b^{-1}, \text { and } \\
& \begin{array}{l}
z w=-(x+y) x y^{-1}=-x^{2} y^{-1} t-y x y^{-1}=-x^{2} y^{-1}-\rho x \\
\quad=-x\left(x y^{-1}+\rho y^{-1} y\right)=-x\left(\rho y^{-1} x+\rho y^{-1} y\right)=-\rho x y^{-1}(x+y)=\rho w z
\end{array}
\end{aligned}
$$

Hence $w, z$ generate the algebra $D_{\left(-a b^{-1}, a+b\right)}$ which therefore must be isomorphic to $D_{(a, b)}$ as desired.

Proposition 1.9. Suppose that $\alpha=\beta \cup(a)$, and that $N$ is a degree $p$ form defined on a vector space $V$ which neutralizes and splits $\beta$. Then then the form $N^{\prime}=N \oplus-a N$ on $V \oplus V$ splits $\alpha$ and neutralizes $\alpha$.

Proof. Suppose that $v, w \in V$. We first show that $N^{\prime}$ splits $\alpha$. Suppose that we have $v, w \in V$ such that $N(v)-a N(w)=0$. We must show in this case that $\alpha=0$. Note that we may assume $N(v), N(w) \neq 0$ since otherwise, using the fact that $N$ splits $\beta$, we would have $\beta=0$ and hence $\alpha=0$ as well. But now we may write

$$
\alpha=\beta \cup(a)=\beta \cup(N(w))+\beta \cup(a)=\beta \cup(a N(w))=\beta \cup(N(v))=0
$$

Now, to see that $N$ neutralizes $\alpha$, let $v, w \in V$. We must show that $\alpha \cup(N(v)-a N(w))=0$. If either $N(v)$ or $N(w)$ are zero, then $\beta=0$ since $N$ splits $\beta$, and hence $\alpha \cup(N(v)-a N(w))=0$ as well. Hence we may assume that $N(w), N(v) \neq 0$. Then we have:

$$
\begin{aligned}
\alpha \cup(N(v)-a N(w)) & =\beta \cup(a) \cup(N(v)-a N(w)) \\
& =\beta \cup\left(N(w) N(v)^{-1} a\right) \cup(N(v)-a N(w)) \\
& =\beta \cup\left(-(-a N(w)) N(v)^{-1}, N(v)-a N(w)\right)
\end{aligned}
$$

By Lemma 1.8, we may then write

$$
\begin{aligned}
\beta \cup\left(-(-a N(w)) N(v)^{-1}, N(v)-a N(w)\right)=\beta & \cup(-a N(w), N(v)) \\
& =\beta \cup(N(v)) \cup\left(-a^{-1} N(w)^{-1}\right)
\end{aligned}
$$

But $\beta \cup(N(v))=0$ since $N$ neutralizes $\beta$, hence $\alpha \cup(N(v)-a N(w))=0$ as desired.

Of course, in the case $p=2$, the Pfister forms give examples of forms which neutralize and split symbols. The contents of the following Proposition were noted by Serre (see [Ser94, page 99]):

Proposition 1.10. Suppose that $p=2$ and $\alpha=\left(a_{1}, \ldots, a_{n}\right) \in k_{n}(F)$ is a symbol. Let $q=\left\langle\left\langle a_{1}, \ldots, a_{n}\right\rangle\right\rangle$ be the corresponding Pfister form. Then $q$ splits and neutralizes $\alpha$.

Proof. By the Milnor conjectures, we have an isomorphism $e_{n}: I^{n}(F) / I^{n+1}(F) \rightarrow$ $k_{n}(F)$ sending $q$ to $\alpha$. If $q$ is isotropic, then since it is a Pfister form, it must be split and in particular represent the 0 class in $I^{n}(F) / I^{n+1}(F)$. It then follows that $\alpha=e_{n}(q)$ is zero as well. 
To see that $q$ neutralizes $\alpha$, suppose that $a=q(v)$, and consider $\alpha \cup(a)=$ $e_{n+1}(q \otimes\langle 1,-a\rangle)$. The form $q \otimes\langle 1,-a\rangle=q \perp-q(v) q$ is isotropic. This is because $q$, being a Pfister form, represents 1 , say $q(w)=1$, and then we may write

$$
(q \perp-q(v) q)(v, w)=q(v)-q(v) q(w)=0 .
$$

But as before, it follows that since $q \otimes\langle 1,-a\rangle$ is a Pfister form, it must in fact be hyperbolic implying that $\alpha \cup(a)=e_{n+1}(q \otimes\langle 1,-a\rangle)=0$ as desired.

Let us now record some applications of these results. The first few of which are well known consequences of previous results in the area.

Theorem 1.11. Suppose that $F$ is $C_{n}$, and let $p=2$. Then every element of $k_{n}(F)=K_{n}^{M}(F) / 2$ is a symbol and $k_{n+1}(F)=0$. In particular, $\operatorname{cd}_{2}(F) \leq n$.

Proof. For the first claim, suppose that $\alpha=\left(a_{1}, \ldots, a_{n}\right)$ and $\beta=\left(b_{1}, \ldots, b_{n}\right)$ are symbols in $k_{n}(F)$. It suffices to show that we may rewrite $\alpha$ and $\beta$ so that $a_{i}=b_{i}$ for $i=1, \ldots, n-1$. We do this by induction on the number of slots that a presentation of $\alpha$ and $\beta$ share. Suppose that a given presentation of $\alpha$ and $\beta$ share $m<n-1$ slots. Without loss of generality, we may suppose that we have $a_{i}=b_{i}$ for $i=1, \ldots, m$ for $m<n-1$. By Proposition 1.10, we may find forms $F, G$ of degree 2 on $2^{n-1}$-dimensional vector spaces $V, W$ which split and neutralize $\left(a_{1}, \ldots, a_{n-1}\right)$ and $\left(b_{1}, \ldots, b_{n-1}\right)$ respectively. Let $\phi$ be the one dimensional form $t^{2} a_{n-1} \in F[t]$. By the $C_{n}$ property, the $2^{n}+1$-dimensional form $\phi \oplus F \oplus G$ has a nontrivial zero, say $a_{n-1} t_{0}^{2}+F(v) a_{n}-G(w) b_{n}=0$ for some $t_{0} \in F, v \in V$ and $w \in W$. If either $F(v)$ or $G(w)$ is zero, then one of the forms $\alpha$ or $\beta$ is trivial (since these forms split $\alpha$ and $\beta$ ), and we are done. Hence we may assume that $F(v), G(w) \neq 0$. But now, we have

$$
\left(a_{1}, \ldots, a_{n}\right)=\left(a_{1}, \ldots, F(v) a_{n}\right)=\left(a_{1}, \ldots, t_{0}^{2} a_{n-1}, F(v) a_{n}\right)
$$

If $t_{0}^{2} a_{n-1}+F(v) a_{n}=0$, it would follow by the definition of Milnor K-theory that $\left(t_{0}^{2} a_{n-1}, F(v) a_{n}\right)=0$ and hence $\alpha=0$. Hence we may assume that this is not the case. By Lemma 1.8 , we may therefore write

$$
\begin{aligned}
\left(t_{0}^{2} a_{n-1}, F(v) a_{n}\right)=\left(-t_{0}^{2} a_{n-1}\left(F(v) a_{n}\right)^{-1}, t_{0}^{2} a_{n-1}\right. & \left.+F(v) a_{n}\right) \\
& =\left(-t_{0}^{2} a_{n-1}\left(F(v) a_{n}\right)^{-1}, G(w) b_{n}\right)
\end{aligned}
$$

Combining this with equation (1), it follows that we may write

$$
\alpha=\left(a_{1}, \ldots, a_{n}\right)=\left(a_{1}, \ldots,-t_{0}^{2} a_{n-1}\left(F(v) a_{n}\right)^{-1}, G(w) b_{n}\right)
$$

and writing $\beta=\left(b_{1}, \ldots, b_{n}\right)=\left(b_{1}, \ldots, b_{n-1}, G(w) b_{n}\right)$, we see that our two new presentations now have $m+1$ slots in common. The claim follows.

For the second assertion, it suffices to show that every symbol $\alpha=$ $\left(a_{1}, \ldots, a_{n+1}\right)$ is trivial in $k_{n+1}(F)$. But via the Milnor conjectures, we may identify $\alpha$ as the $e_{n+1}$ invariant of the $(n+1)$-fold Pfister form $\left\langle\left\langle a_{1}, \ldots, a_{n+1}\right\rangle\right\rangle$, which has dimension $2^{n+1}$. Since $F$ is $C_{n}$, it follows that this Pfister form is isotropic and hence hyperbolic. Consequently $\alpha$, its $e_{n+1}$-invariant, must vanish. 
We next recall a consequence of the work of Merkurjev and Suslin:

Theorem 1.12. Let $F$ be $C_{2}$ then $k_{3}(F)=K_{3}^{M} / p=0$, and so $\operatorname{cd}(F) \leq 2$.

Proof. Let $\alpha=(a, b, c) \in k_{3}(F)$. Let $D=D_{(a, b)}$ be the symbol algebra associated to $(a, b)$, and let $N_{D}$ be its reduced norm. By Example 1.6, $N_{D}$ splits and neutralizes $(a, b)$. Therefore, by Proposition 1.9, the form $N=N_{D} \oplus-c N_{D}$ splits and neutralizes $\alpha$. But note that this form has $2 p^{2}>p^{2}$ and degree $p$. Since $F$ is $C_{2}$, it follows that it is isotropic and therefore $\alpha=0$ as desired.

Theorem 1.13. Let $F$ be $C_{3}$. Then $K_{4}^{M} / 3=0$, and so $\operatorname{cd}_{3}(F) \leq 3$.

Proof. If $A$ is the Albert algebra for $(a, b, c)$ then $N_{A} \oplus-d N_{A}$ is a $2 \cdot 3^{3}=54$ dimensional form splitting and neutralizing $(a, b, c, d)$. since $54>27$ we are done.

Theorem 1.14. Let $F$ be $C_{4}$. Then $K_{5}^{M} / 3=0$ and so $\operatorname{cd}_{4}(F) \leq 4$.

Proof. If $A$ is the Albert algebra for $(a, b, c)$ then $N=N_{A} \oplus-d N_{A}$ is a $2 \cdot 3^{3}=54$ dimensional form splitting and neutralizing $(a, b, c, d)$, and $N^{\prime}=N \oplus-e N$ is a 108 dimensional form splitting and neutralizing $(a, b, c, d, e)$. Since $108>$ $81=3^{4}$ we are done.

Theorem 1.15. Let $F$ be $C_{n}$. Then

(1) $\operatorname{cd}_{2}(F) \leq n$,

(2) $\operatorname{cd}_{3}(F) \leq\left\{\begin{array}{cc}n & \text { if } n \leq 4 \\ \left\lceil(n-3)\left(\log _{2}(3)\right)+3\right\rceil & \text { otherwise, }\end{array}\right.$

(3) $\operatorname{cd}_{p}(F) \leq\left\{\begin{array}{cc}n & \text { if } n \leq 2 \\ \left\lceil(n-2)\left(\log _{2}(p)\right)+1\right\rceil & \text { otherwise. }\end{array}\right.$

For example, if $F$ is $C_{3}$ then $\operatorname{cd}_{5}(F), \operatorname{cd}_{7}(F) \leq 4$. Note that in particular, the $p$-cohomological dimension is bounded linearly with respect to the Diophantine dimension, with the slope $\log _{2}(p)$.

Proof. The first part, corresponding to $\mathrm{cd}_{2}$ follows from Theorem 1.11

For $\mathrm{cd}_{3}$, the cases with $n \leq 4$ follow from Propositions 1.13 and 1.14 respectively. In general, to check whether or not $\operatorname{cd}_{3}(F) \leq m$, write a degree $m+1$ symbol in $K_{m+1}^{M} / 3$ as $\alpha=(a, b, c) \cup\left(d_{1}, \ldots, d_{m-2}\right)$. We wish to find a criterion on $m$ which will guarantee that $\alpha=0$. This works as follows: if $N_{A}$ is the norm form for the first Tits process Albert algebra $A$ defined by $(a, b, c)$, then inductively applying Proposition 1.9 we obtain a form $N$ which splits $\alpha$ in $27 \cdot 2^{m-2}$ variables of degree 3 . In particular, by the $C_{n}$ property, we find that this form is isotropic, and hence $\alpha$ is split when $27 \cdot 2^{m-2}>3^{n}$ or $2^{m-2}>3^{n-3}$. But this translates to $m>(n-3)\left(\log _{2}(3)\right)+3$, since $\log _{2} 3$ is irrational, this is equivalent to saying $m \geq\left\lceil\left(n-3\left(\log _{2}(3)\right)+3\right\rceil\right.$.

The general case of $\mathrm{cd}_{p}$ follows much like the case of $\mathrm{cd}_{3}$ above, considering a symbol $\alpha=(a, b) \cup\left(c_{1}, \ldots, c_{m-1}\right)$, starting with Theorem 1.12 and applying Proposition 1.9 to obtain a form $N$ of degree $p$ and dimension 
$p^{2} \cdot 2^{m-1}$ splitting $\alpha$. Again, in this case, the $C_{n}$ property guarantees that $\alpha$ is split in case $2^{m-1}>p^{n-2}$. The result follows.

Remark 1.16. Although it is a weaker result, it is interesting to note that one may associate an "obvious form" which splits a given symbol. In particular if $\alpha=\left(a_{1}, \ldots, a_{n}\right) \in k_{n}(F)$, then the form $a_{1} t_{1}^{p}+\cdots+a_{n} t_{n}^{p}$ splits $\alpha$.

Proof. We induct on $n$, the case $n=1$ being trivial and the case $n=2$ following from the fact that $N_{D_{(a, b)}}$ when restricted to the subspace $F x+F y$ is exactly the form $a t^{p}+b s^{p}$. For the general induction step, suppose that $\sum a_{i} r_{i}^{p}=0$ for some choice of $r_{i} \in F$, and set $u=\sum_{i=0}^{n-2} a_{i} r_{i}^{p}$. Then $-u=$

$a_{n-1} r_{n-1}^{p}+a_{n} r_{n}^{p}$. By Lemma1.8, we may write $\left(a_{n-1}, b_{n-1}\right)=(-u, v)$ for some $v \in F^{*}$. But therefore we may write $\alpha=\left(a_{1}, \ldots, a_{n-2},-u, v\right)$. Considering the form $\left(a_{1}, \ldots, a_{n-2},-u\right)$ we find that by $\sum_{i=1}^{n-2} a_{i} r_{i}^{p}+(-u) 1=0$, the induction hypothesis implies $\left(a_{1}, \ldots, a_{n-2},-u\right)=0$ and so $\alpha=\left(a_{1}, \ldots, a_{n-2},-u\right) \cup(v)=0$ as well.

\section{Appendix: Norm Forms ON POWER ASSOCIATIVE ALgEBRAS}

Recall that an algebra $A$ is called power associative if all associators of the form $\left\{a^{i}, a^{j}, a^{k}\right\}$ vanish, or equivalently, if for every $a \in A$, the subalgebra $F[a]$ generated by $a$ is associative and commutative. We say that $A$ is strictly power associative if for every field extension $L / F$, the algebra $A_{L}=A \otimes_{F} L$ is power associative. Note that in case $F$ has characteristic not equal to 2 , by linearizing associator relations such as $\{x, x, y\}$, one sees that every power associative algebra is automatically strictly power associative.

Let $A$ be a unital finite dimensional strictly power associative $F$-algebra. We now recall the definition of the reduced norm function on $A$. Set $R=$ $F\left[A^{*}\right]$ to be the ring of polynomial functions on $A$. Consider the generic element $x \in A_{R}$, defined as follows: if $a_{1}, \ldots a_{n}$ is a basis for $A$ and $f_{1}, \ldots, f_{n} \in$ $A^{*} \subset R=F\left[f_{1}, \ldots, f_{n}\right]$ is the corresponding dual basis, then $x=\sum a_{i} f_{i}$. In a coordinate free way, we may also write $x$ as the identity map, thought of as an element of $\operatorname{Hom}_{F}(A, A)=A \otimes A^{*} \subset A \otimes R=A_{R}$. We may now consider the subalgebra $R[x] \subset A_{R}$. Since $A_{R}$ is a finitely generated module and $R$ is Noetherian, it follows that $R[x]$ is finitely generated $R$-module and hence an integral extension. Let $m(T)=m_{0}+m_{1} T+\cdots+m_{r-1} T^{r-1}+T^{r} \in R[T]$ be the minimal polynomial of $T$. Since $m(x)$ is the zero polynomial function on $A$, it follows that for any $a \in A, a$ is a root of function

$$
\chi_{a}(T)=m_{0}(a)+m_{1}(a) T+\cdots+m_{r-1}(a) T^{r-1}+T^{r}
$$

which we call the reduced characteristic polynomial of $a$. We define the reduced norm of $a, N_{A}(a)$ to be $m_{0}(a)$. The reduced norm function is the function $N_{A}=m_{0} \in R$. We define the adjunct of $a$, defined by $\operatorname{adj}_{A}(a)$ to be $m_{1}(a)+m_{2}(a) a+\cdots m_{r-1}(a) a^{r-2}+a^{r-1}$. We then have $a\left(\operatorname{adj}_{A}(a)\right)=N_{A}(a)$. Note that since these coincide with the standard characteristic and minimal 
polynomials of the $R$-algebra $R[x]$ acting on itself, we have the familiar identity $\chi_{a}(T)=N_{A}(T-a)$.

We say that a power associative finite dimension $F$-algebra $A$ has degree $d$ if $\chi_{A}$ (and hence also $N_{A}$ ) is a degree $d$ polynomial function.

Recall that a nonassociative algebra $A$ is called division in case left and right multiplication by every nonzero element $a \in A$ are both bijective. We say that $A$ is principally division, if for every $a \in A$, the subalgebra $F[a]$ generated by $a$ is division. We say that $a \in A$ is invertible if there exists $b$ such that $a b=1=b a$.

Lemma 2.1. Suppose that $A$ is a power associative F-algebra of prime degree $p$. Then $A$ is principally division if and only if $N_{A}$ is anisotropic.

Proof. Suppose $N_{A}$ is anisotropic and let $a \in A$. We must show that $F[a]$ is division. Let $b \in F[a] \subset A$ with $b \neq 0$. Since $N_{A}(b) \neq 0$, we may write $b\left(N_{A}(b)\right)^{-1}\left(\operatorname{adj}_{A}(b)\right)=1$, and since $\operatorname{adj}_{A}(b) \in F[b] \subset F[a]$, it follows that $b$ is invertible in $F[a]$. Therefore $F[a]$ is division.

Conversely, if $A$ is principally division, we wish to show that for all $a \in A \backslash 0, N_{A}(a) \neq 0$. Let $m_{a}(t)$ be the minimal polynomial of $a$. It follows from [Jac59], that $m_{a}(t)$ and $\chi_{a}(t)$ have the same prime factors. Since $F[a]=$ $F[t] /\left(m_{a}(t)\right)$ is a division algebra, it follows that $m_{a}(t)$ is irreducible, and in particular, not divisible by $t$. But therefore $t$ cannot divide $\chi_{a}(t)$ as well. It therefore follows that $N_{A}(a)$, being (up to a sign) the constant coefficient of $\chi_{a}(t)$ is also nonzero, as desired.

Lemma 2.2. Suppose $A$ is a finite dimensional alternative algebra. Then $A$ is principally division if and only if it is division.

Proof. Certainly if $A$ is division, it is also a domain. Since each subalgebra $F[a]$ is then a finite dimensional domain, they are domains, and hence $A$ is principally division.

For the converse, suppose that $A$ is principally division, and let $a \in A$. We wish to show that left multiplication by $a$ gives an bijection from $A$ to itself. The proof that right multiplication also has this property follows from the same argument.

To see this, we start from the fact that $F[a]$ is a field, and, using the fact that $A$ is left alternative, left multiplication gives $A$ the structure of a vector space over $F[a]$. But scalar multiplication by a nonzero scalar is automatically an isomorphism of $A$ with inverse given by $a^{-1}$.

Lemma 2.3. Let $A$ be a first Tits process Albert algebra over F. Then $A$ is division, if and only if it is principally division.

Proof. Clearly if $A$ is division it is principally division. For the converse, suppose that $A$ is not division. By [Jac68, Chapter 9, Theorem 20], $A$ is split, and hence must contain the algebra $M_{n}(F)^{+}$. But it follows that $A$ is therefore not principally division, since, for example, it contains nilpotent elements. 


\section{REFERENCES}

[Ax65] James Ax. A field of cohomological dimension 1 which is not $C_{1}$. Bull. Amer. Math. Soc., 71:717, 1965.

[Jac59] N. Jacobson. Some groups of transformations defined by Jordan algebras. I. J. Reine Angew. Math., 201:178-195, 1959.

[Jac68] Nathan Jacobson. Structure and representations of Jordan algebras. American Mathematical Society Colloquium Publications, Vol. XXXIX. American Mathematical Society, Providence, R.I., 1968.

[KMRT98] Max-Albert Knus, Alexander Merkurjev, Markus Rost, and Jean-Pierre Tignol. The book of involutions. American Mathematical Society, Providence, RI, 1998. With a preface in French by J. Tits.

[LMS07] Nicole Lemire, Ján Mináč, and John Swallow. Galois module structure of Galois cohomology and partial Euler-Poincaré characteristics. J. Reine Angew. Math., 613:147-173, 2007.

[PR96] Holger P. Petersson and Michel L. Racine. An elementary approach to the SerreRost invariant of Albert algebras. Indag. Math. (N.S.), 7(3):343-365, 1996.

[Ser94] Jean-Pierre Serre. Cohomologie galoisienne, volume 5 of Lecture Notes in Mathematics. Springer-Verlag, Berlin, fifth edition, 1994.

[Voe03] Vladimir Voevodsky. Motivic cohomology with Z/2-coefficients. Publ. Math. Inst. Hautes Études Sci., (98):59-104, 2003. 